\title{
Modelling of Dynamic Recrystallisation of 316L Stainless Steel using a Systems Approach
}

\author{
M.F. Abbod ${ }^{1, a}$, M. Mahfouf ${ }^{1, b}$, D.A. Linkens ${ }^{1, c}$ and C.M. Sellars ${ }^{2, d}$ \\ Institute for Microstructural and Mechanical Process Engineering: The University of Sheffield \\ ${ }^{1}$ Department of Automatic Control and Systems Engineering, \\ ${ }^{2}$ Department of Engineering Materials, \\ The University of Sheffield, Sheffield, UK \\ Email: ${ }^{a}$ M.F.Abbod@shef.ac.uk, ${ }^{b}$ M.Mahfouf@shef.ac.uk, ${ }^{\circ}$ D.Linkens@shef.ac.uk, \\ dC.M.Sellars@shef.ac.uk
}

Keywords: 316L stainless steel, dynamic recrystallisation, constitutive equation, Modelling, prediction.

\begin{abstract}
Dynamic recrystallisation (DRX) is an important aspect for industrial applications in hot metal working. Although DRX has been known for more than thirty years, its mechanisms have never been precisely investigated, in part because it was not readily possible to make local texture measurements. In the present work, the material behaviour during DRX is investigated and modelled based on the microstructure of 316L stainless steel. The developed model is based on a constitutive equation Modelling technique which incorporates the strain, strain rate and instantaneous temperature for predicting the flow stress of material being deformed under hot conditions.
\end{abstract}

\section{Introduction}

In metallurgy, dynamic recrystallisation (DRX) is a softening process that takes place during metal deformation at elevated temperatures. This softening is observed in a large number of metals and alloys and for numerous deformation processes. Careful analyses of the deformation behaviour, including microstructural investigations, have shown that the softening process involves discontinuous formation of new grains within the deformed matrix. During deformation, as the grains develop by nucleation and growth, the average dislocation density drops, leading to significant softening. This type of dynamic recrystallisation is associated with low or medium stacking fault energy metals (copper, silver, nickel, the austenite phase of conventional steels, and austenitic stainless steels).

In this paper, a constitutive equation model has been developed for the prediction of the flow stress based on the deformation conditions, such as the strain, strain rate and the instantaneous temperature. The majority of laboratory rolling simulations use constant mean values of temperature and strain rate; i.e. constant Zener-Hollomon parameter (Z). However, in industrial rolling there are variations in strain rate and temperature that can produce deviations from equation of state conditions. Such transients in stress-strain relationships and microstructure can significantly affect subsequent processing and final properties. Industry now recognises that it must understand and incorporate transient behaviour if existing physically based models are to become truly predictive tools. This work aims to investigate the effects of controlled changes in strain rate on the instantaneous flow stress of commercial purity austenitic stainless steel (316L) using plane strain compression (PSC) testing.

\section{Dynamic Recrystallisation}

During deformation, work is carried out on the material which implies the storage of dislocations in the original structure. At the same time as work-hardening increases the dislocation density, the softening process of dynamic recovery takes place, diminishing the dislocation density (the more 
dislocations there are, the faster the recovery rate) [1]. Dynamic recovery is a thermally activated process and strain rate dependent: when the temperature rises, the time necessary for this process to occur is lower. This is the reason why the stress-strain curve is very dependent on the strain rate at high temperatures. The work hardening may be athermal, but dynamic recovery occurs from the beginning of the curve, and this explains why the first part of the stress-strain curve is temperature dependent. This is schematically shown in Fig. 1, where the microstructural evolution is illustrated together with the corresponding stress-strain curves.

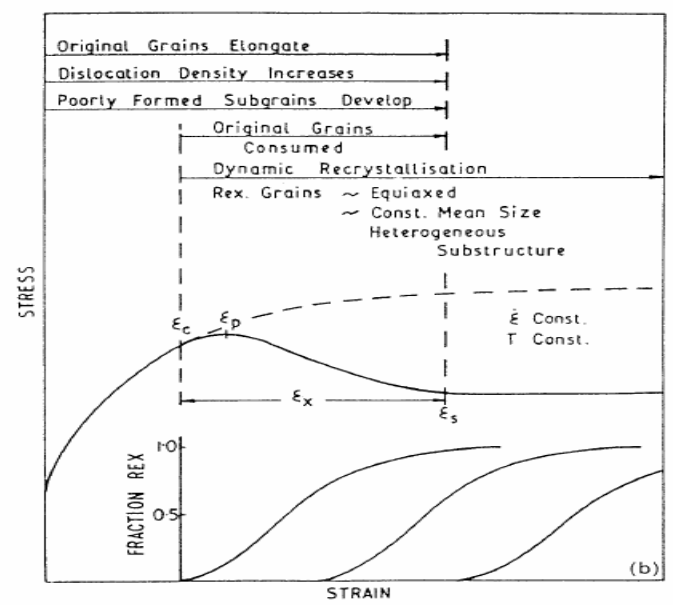

Fig 1. Schematic evolution of microstructural changes during deformation.

\section{L Stainless Steel Alloy Modelling}

In this study, an austenitic 316L stainless steel was selected for the investigation of the hot deformation behavior of a low stacking fault energy FCC metal. The choice of 316L stainless was motivated by the aim of gaining a better understanding of the occurrence of DRX in austenitic alloys. Furthermore, austenitic stainless steel alloys have relatively low stacking fault energy and have been investigated extensively for more than thirty years [2-4].

The chemical composition of the 316L stainless steel used in this investigation is given in Table 1 . The commercial composition material has a stabilized microstructure with a grain size of about $100 \mu \mathrm{m}$.

\begin{tabular}{rrrrrrr}
\hline & $C r$ & $N i$ & $C$ & $M n$ & $S i$ & $M o$ \\
\hline $\mathrm{wt} \%$ & 16.7 & 12.2 & 0.024 & 1.5 & 0.29 & 2.63 \\
\hline
\end{tabular}

Table 1 Composition of the $316 \mathrm{~L}$ stainless steel used in the present work.

Plane strain compression (PSC) specimens with dimensions 50x30x10 mm were machined from the as-received material so that test deformation is parallel to the prior rolling direction. All specimens were coated with a thin film of DAG-2626 lubricant, which was allowed to dry before testing. PSC tests were carried out on a computer controlled servohydraulic machine. The specimens were heated to $1025^{\circ} \mathrm{C}$ and held for 60 seconds. They were then transferred to the test furnace at $1025^{\circ} \mathrm{C}$ and then deformed to the desired strains using constant or variable strain rates in the range $0.5,5.0$ and $50 \mathrm{~s}^{-1}$. The specimens were water quenched immediately after deformation.

The PSC tests were utilised to obtain equivalent true stress/equivalent true strain curves for different test conditions as illustrated in Table 2 cases 1-3.

Furthermore, previous researchers' data [5-6] (Table 2) were utilised to develop the model based on calculating the stresses at a strain of $0.1, \sigma_{0.1}$, and steady state $\sigma_{\mathrm{ss}}$. These stresses were read directly from the curves and were plotted against $\ln \dot{\varepsilon}$ (actual value for the strain of interest) for each point of strain and instantaneous temperature of deformation in order to interpolate the strain rates for constant stresses. The instantaneous temperatures during deformation were calculated using a finite difference 
package based on the dimensions of the samples and the deformation conditions [7]. The temperature rise during deformation is shown in Fig. 2 for the different deformation conditions.

\begin{tabular}{c|cccc}
\hline case & Strain & Strain rate $(1 / \mathrm{s})$ & Temperature $\left({ }^{\circ} \mathrm{C}\right)$ & Author \\
\hline 1 & 1 & 0.5 & 1025 & \\
2 & 1 & 5 & 1025 & Abbod \\
3 & 1 & 50 & 1025 & \\
\hline 4 & 1.4 & 5.24 & 950 & \\
5 & 1.4 & 0.05 & 1025 & \\
6 & 1.4 & 0.5 & 1025 & Barbosa [6] \\
7 & 1.4 & 5 & 1025 & \\
8 & 1.4 & 6.78 & 1100 & \\
\hline 9 & 1.4 & 0.05 & 950 & \\
10 & 1.4 & 0.5 & 950 & Tanaka [5] \\
11 & 1.4 & 5 & 950 & \\
\hline
\end{tabular}

Table 2: Deformation conditions of the PSC tests.

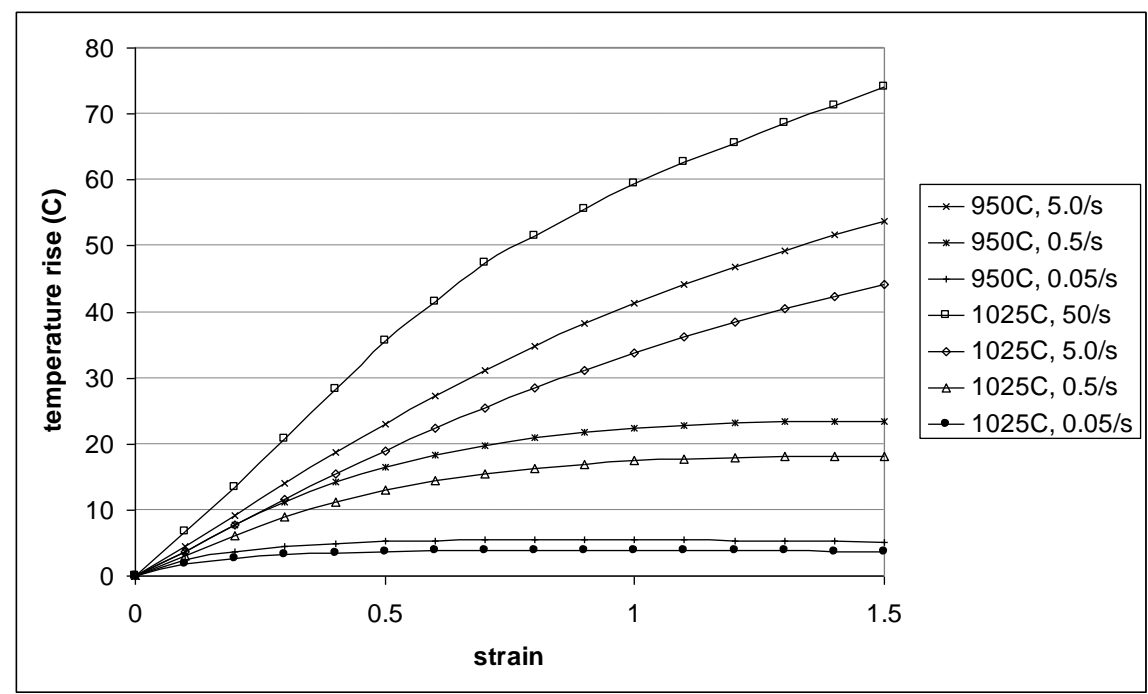

Fig 2: Temperature rise during deformation.

Over the whole stress ranges, the following general equation was used:

$\sigma=\frac{\sinh ^{-1}(\mathrm{Z} / \mathrm{A})^{(1 / \mathrm{n})}}{\alpha}$

Values for $\alpha, \mathrm{n}$ and A were obtained from the stress/strain curves, and can be summarized by the following equations:

$$
\begin{aligned}
& \mathrm{Q}=460 \mathrm{~kJ} / \mathrm{mol} \\
& \varepsilon_{\mathrm{p}}=0.473 \mathrm{~d}_{0}^{0.02863} \mathrm{Z}^{-0.0353} \\
& \varepsilon_{\mathrm{ss}}(\text { isothermal })=0.4691 \mathrm{Z}^{0.0241} \\
& \sigma_{0}=\frac{\sinh ^{-1}(\mathrm{Z} / 1.0 \mathrm{E} 17)^{(1 / 12.98476)}}{0.015218} \\
& \sigma_{0.1}=\frac{\sinh ^{-1}(\mathrm{Z} / 1.0 \mathrm{E} 17)^{(1 / 1.737494)}}{0.016068} \\
& \sigma_{\mathrm{e}}=\frac{\sinh ^{-1}(\mathrm{Z} / 1.0 \mathrm{E} 17)^{(1 / 4.844028)}}{0.008079} \\
& \sigma_{\mathrm{ss}}=\frac{\sinh ^{-1}(\mathrm{Z} / 1.0 \mathrm{E} 17)^{(1 / 4.763623)}}{0.00966}
\end{aligned}
$$


The final equivalent stress is calculated using equation 8 for low strain while the effect of the softening process is expressed by equation 9 for high strain.

$$
\begin{array}{ll}
\sigma^{\prime}=1.0\left\{\sigma_{0}+\left(\sigma_{\mathrm{e}}-\sigma_{0}\right)\left[1-\exp \left(-\mathrm{C}^{\prime} \varepsilon\right)\right]^{0.5}\right\} & \left(\varepsilon<0.7 \varepsilon_{\mathrm{p}}\right) \\
\left.\sigma=\sigma^{\prime}-1.0\left(\sigma_{\mathrm{e}}-\sigma_{\mathrm{ss}}\right)\left\{1-\exp \left[-0.49\left(\frac{\varepsilon-0.7 \varepsilon_{\mathrm{p}}}{\varepsilon_{\mathrm{ss}} \text { (isotherma) }-\varepsilon_{\mathrm{p}}}\right)\right)^{1.4}\right]\right\} & \left(\varepsilon \geq 0.7 \varepsilon_{\mathrm{p}}\right)
\end{array}
$$

where $C^{\prime}=\left(\frac{\sigma_{0.1}-\sigma_{0}}{\sigma_{e}-\sigma_{0}}\right)^{2}$

Fig. 3 shows the experimental and predicted flow stress as a function of strain for the defined deformation conditions. Model validation was carried out for constant Zener-Hollomon deformation conditions given in Table 3 and results are shown in Fig 4.

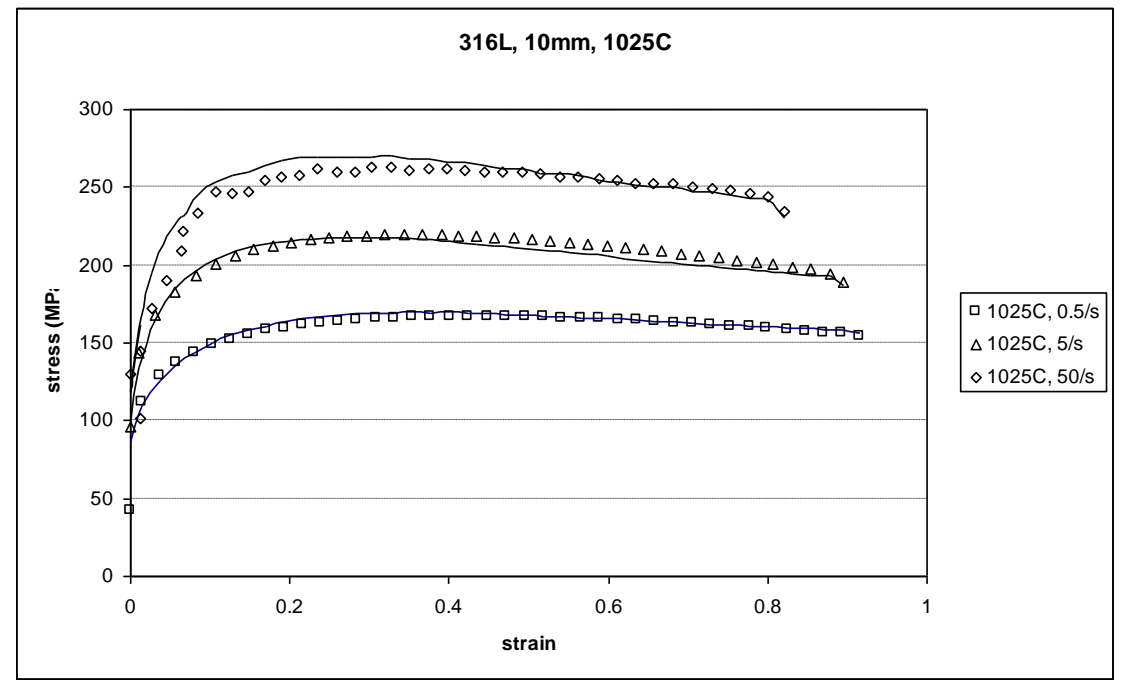

Fig 3. Experimental and modelled data for PSC tests.

\begin{tabular}{c|cccc}
\hline case & Strain & Strain rate $(1 / s)$ & Temperature $\left({ }^{\circ} \mathrm{C}\right)$ & $Z(1 / s)$ \\
\hline 1 & 1.2 & 0.5 & 1025 & $1.74731 \mathrm{E}+18$ \\
2 & 1.2 & 5 & 1025 & $1.74731 \mathrm{E}+19$ \\
3 & 1.2 & 50 & 1025 & $1.74731 \mathrm{E}+20$ \\
\hline
\end{tabular}

Table 3: Deformation conditions constant Zener-Hollomon tests.

The constant Zener-Hollomon conditions are achieved by changing the strain rate in order to keep Z constant due to the temperature rise, Fig. 4, does not appear to show any evidence of mechanical transients in either material, for any of the rates of change in strain rate used. In all cases, the flow stress appears to have reached the level expected for constant strain rate conditions by the end of the test. Barraclough and Sellars [3] suggested that there would be no cumulative effect on flow stress from a strain rate change, providing the rate of change was less than some limiting value, $\mathrm{H}_{\mathrm{lim}}$, given by:

$$
\mathrm{H}_{\lim }=\frac{\Delta \log \mathrm{Z}}{\Delta \varepsilon}=\left(\frac{\mathrm{d} \log \mathrm{Z}}{\mathrm{d} \varepsilon}\right)_{\lim }
$$

Below this rate, flow stress would depend only on the instantaneous strain rate, and a mechanical equation of state would apply. No transient is evident for any of the rates of change used, 0.5, 0.34 and 0.055 for cases 1-3 respectively (Fig. 5), suggesting a very low experimental value of $\mathrm{H}$ are bellow the $\mathrm{H}_{\text {lim }}$ in austenitic stainless steels.

However, it would be necessary to plot the expected flow stress paths for changing strain rates, assuming an equation of state is obeyed, and compare these with the experimental results to confirm 
that there are no transients. This requires the derivation of an equation for the material and deformation conditions used.

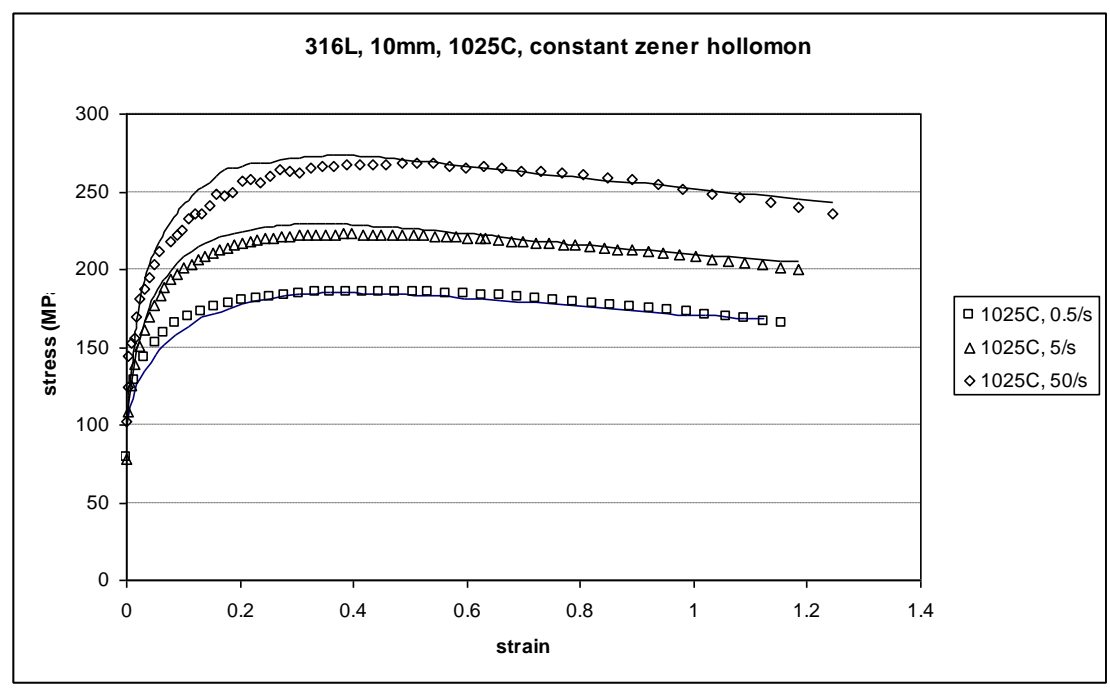

Fig 4. Validation of the constitutive equations model for PSC tests.

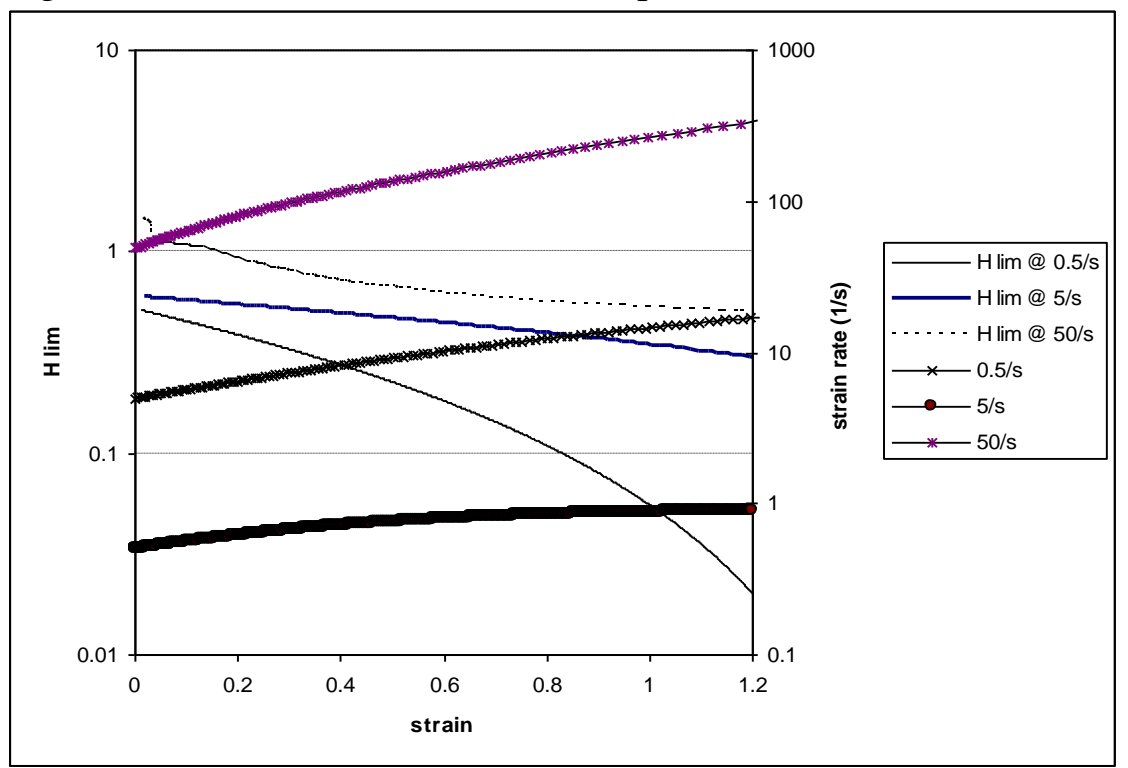

Fig 5. $\mathrm{H}_{\mathrm{lim}}$ and strain rate changes for constant Zener-Hollomon testing.

\section{Model Validation}

For unseen data, the model has been utilised to predict the behaviour of different deformation conditions which have not been used in the model fitting stage. Comparison of experimental and predicted results for flow stress evolution of a commercial purity $316 \mathrm{~L}$ stainless steel $\left(\mathrm{d}_{0}=100 \mu \mathrm{m}\right)$ during hot deformation under PSC test deformation conditions is presented in Fig. 6 for the steady state and peak stress respectively. The results calculated with the developed model under different deformation conditions via different researchers show good agreement with the experimental data.

\section{Conclusions}

A DRX constitutive model has been developed incorporating experimental testing conditions and validated on constant Zener-Hollomon deformation conditions. The model was further validated on experimental work from the literature which is based on the peak and steady state flow stresses. The flow stresses computed from the recrystallisation kinetics model reproduce relatively well the shapes of the experimental curves. The shape of the first peak is mainly controlled by the work hardening law 
and by the kinetics of the first recrystallisation wave. The steady-state flow stress is mainly controlled by the recrystallisation kinetics. Future work will concentrate on the mechanical equation of state for the material and deformation conditions used which should be derived to compare changing strain rate flow stresses with predicted curves, to check for evidence of transients and deviations form the equation of state.
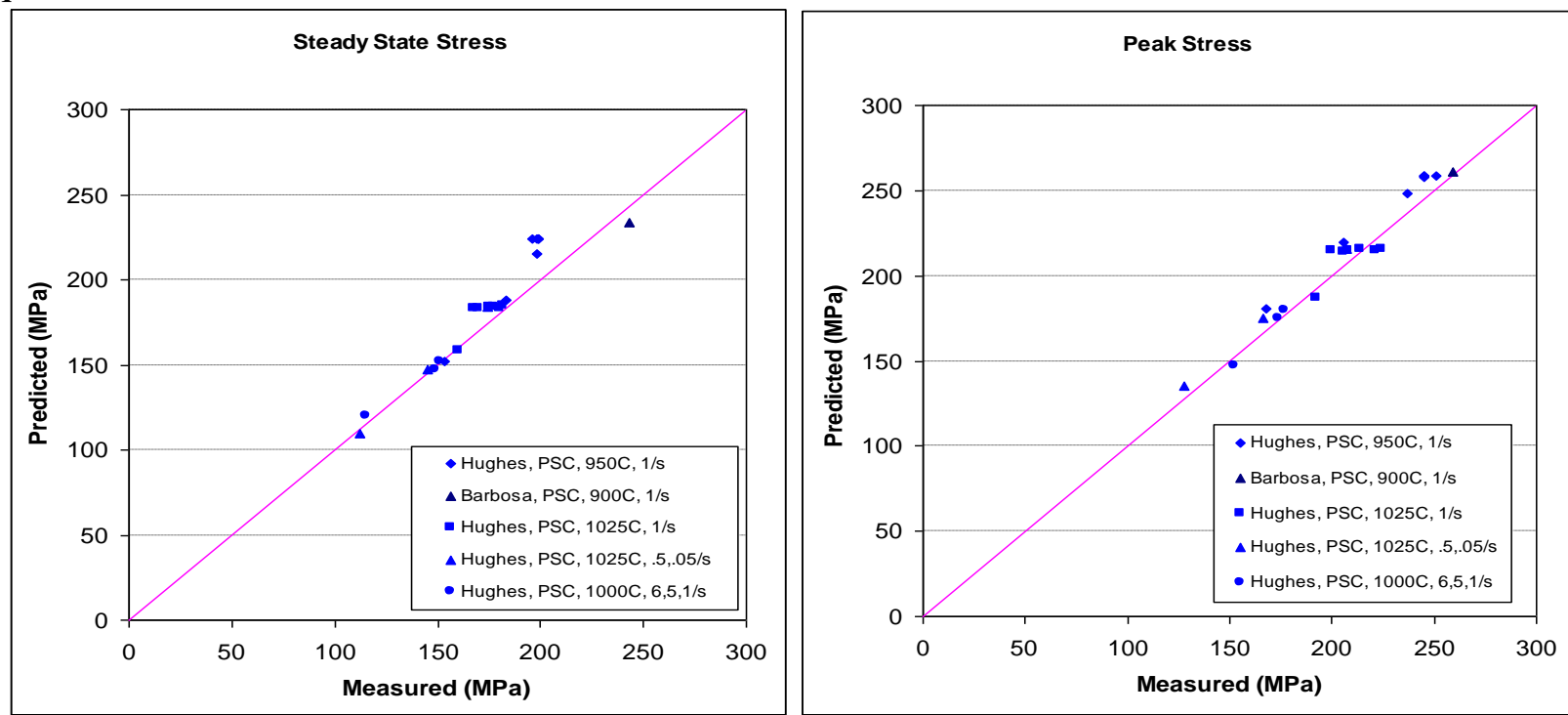

Fig. 6. Comparison of experimental and predicted results for peak and steady state flow stress.

\section{Acknowledgment}

The authors gratefully acknowledge the UK EPSRC (Engineering and Physical Sciences Research Council) for their financial support under grant number GR/R70514/01.

\section{References}

[1] C.M. Sellars: Modelling of structural evolution during hot working processes, Proc. RIS $\emptyset$ international symposium on annealing processes: recovery, recrystallisation and grain growth (1986), pp. 167-187.

[2] D.R. Barraclough: Hot working and recrystallisation of a stainless and a low alloy steel. Ph.D. thesis, University of Sheffield, U.K (1974).

[3] D.R. Barraclough and C.M. Sellars: Static recrystallisation and restoration after hot deformation of type 304 stainless steel. Metal Science, Vol. 13 (1979), pp. 257-267.

[4] N.D. Ryan and H.J. McQueen: Comparison of dynamic softening of 301, 304, 316 and 317 stainless steels. High Temperature Technology, Vol. 8, No. 3 (1990), pp. 185-200.

[5] A. Tanaka: Effect of non-isothermal multipass hot deformation on strength and recrystallisation kinetics, Thesis (M.Phil.), University of Sheffield, Dept. of Metallurgy, (1987).

[6] R.A.N. Barbosa: Simulation of hot working of austenitic stainless steels. Thesis (Ph.D.), University of Sheffield, Dept. of Metallurgy (1985).

[7] R.J. Hand, S.R. Foster and C.M. Sellars: Temperature Changes during Hot Plane Strain Compression Testing. Mat. Sci. and Tech. Vol 16 (2000), 442-450.

[8] R.A.N. Barbosa and C.M. Sellars: Static Recrystallisation of Type 316L Stainless Steel under Hot Working Conditions, Recrystallisation'92, Proc. Int. Conf. on Recrystallisation and Related Phenomena, San Sebastian, Spain, 1992, eds. M. Fuentes and J. Oil Sevillano, Trans Tech Publications, Switzerland (1992), pp. 461-466.

[9] K.E. Hughes, K.D. Nair and C.M. Sellars: Temperature and Flow Stress during the Hot Extrusion of Steel. Metals Tech. (1974), pp. 161-169. 